\title{
Phytoremediation: le piante per bonificare i siti contaminati
}

\author{
Vincenzo Marino $^{1}$ \\ ${ }^{1}$ University of Salerno
}

\begin{abstract}
La problematica dei siti contaminati è di grande attualità e assume un'importanza estremamente rilevante nell'ambito del rischio ambientale e della salute pubblica. I contaminanti del suolo, infatti, possono essere caratterizzati da alti livelli di tossicità, di persistenza nell'ambiente e da una certa mobilità che può portare alla contaminazione delle acque sotterranee e alla catena alimentare, fino a diventare fonte di pericolo per la salute umana. I siti contaminati necessitano, quindi, di interventi di bonifica che si differenziano in funzione delle caratteristiche sito-specifiche, di fattori economici e degli obiettivi della bonifica. Tra le diverse tecniche si inserisce la Phytoremediation, che sfrutta le proprietà ecofisiologiche di particolari tipi di piante per ridurre le concentrazioni di inquinanti nel suolo. Il presente elaborato tratta un caso studio in cui tale tecnica è stata adottata con particolare efficacia per bonificare un sito contaminato da metalli pesanti $(\mathrm{Cd}, \mathrm{Hg}, \mathrm{Zn})$.
\end{abstract}

\section{Una tecnica di bonifica alternativa: la Phytoremediation}

La bonifica dei siti contaminati è uno dei temi più rilevanti nell'ambito degli interventi di recupero e di risanamento ambientale, pertanto l'identificazione, la caratterizzazione ed il recupero di aree contaminate costituiscono uno dei settori di attività di tutela ambientale di maggiore interesse. In 
queste aree è necessario intervenire per mettere in sicurezza il sito e successivamente per eseguire attività volte a ridurre il contenuto nel suolo di contaminanti. Per evitare che le concentrazioni di contaminanti siano superiori ai limiti normativi, che in Italia sono regolamentati dal D. Lgs. 152/06 al titolo V parte IV, sono state sviluppate diverse tecnologie e metodi per rimuoverli dai terreni inquinati. Molti di questi metodi includono la rimozione fisica del suolo nelle discariche o l'estrazione attraverso mezzi chimici o fisici ${ }^{1}$. Diversi metodi fisici e chimici usati a questo scopo soffrono di gravi limitazioni come costi elevati, alterazione delle proprietà del suolo e disturbo della microflora nativa del suolo ${ }^{2}$. Al contrario, la Phytoremediation è una strategia di riabilitazione efficiente, economica ed ecologica.

\section{La rimozione di metalli pesanti mediante Fitoestrazione}

La Phytoremediation è una tecnica di biorisanamento che utilizza piante per rimuovere, degradare e immobilizzare contaminanti sia organici che inorganici dal suolo ${ }^{3}$. In funzione delle caratteristiche del suolo, della tipologia di pianta utilizzata per il processo e del contaminante da trattare è possibile identificare diversi meccanismi di Phytoremediation ${ }^{4}$. La contaminazione del suolo a causa dei metalli pesanti è una grave minaccia per l'umanità ed è una preoccupazione che si estende a livello globale ${ }^{5}$. È noto che i metalli pesanti come piombo $(\mathrm{Pb})$, mercurio $(\mathrm{Hg})$, cromo $(\mathrm{Cr})$ e alcuni metalloidi come arsenico (As), selenio ( $\mathrm{Se}$ ) e antimonio ( $\mathrm{Sb}$ ) sono altamente tossici per l'uomo anche a piccole dosi e quindi, alcune di esse sono classificate tra le sostanze più pericolose 67. Tra i diversi meccanismi di Phytoremediation, il più indicato per la bonifica di siti contaminati da metalli pesanti è la Fitoestrazione che prevede l'accumulo di contaminanti nella massa vegetale, stelo e foglie (Fig.1), che dovrà poi essere trattata o rimossa. La Fitoestrazione si basa sull'uso di piante ad alta capacità di accumulo dette iperaccumulatrici, che sono in grado di tollerare e accumulare alte concentrazioni di metalli. Iperaccumulatori ideali richiedono le caratteristiche di radici profonde, crescita rapida e un' elevata quantità di biomassa. ${ }^{8}$. 
Il caso studio analizzato riporta i risultati di un'applicazione della tecnologia di Fitoestrazione in un'area della zona industriale per risanare un terreno contaminato da metalli pesanti a causa di un'intesa attività industriale ${ }^{9}$. Innanzitutto, l'area oggetto di studio è stata suddivisa in due poligoni (SP3 e FS4) e sono state condotte indagini preliminari in 7 punti di campionamento al fine di valutare le proprietà del suolo e la contaminazione. Sono stati riscontrati valori di concentrazione di 3,41 mg/kg di Cd nel poligono SP3 e 3,24 mg/kg di Cd in FS4 (soglia di concentrazione di contaminazione $2 \mathrm{mg} / \mathrm{kg}$ ); la concentrazione iniziale di $\mathrm{Hg}$, invece, è risultata pari a $1,47 \mathrm{mg} / \mathrm{kg}$ per il poligono SP3 e 1,46 mg/kg per FS4 (soglia di concentrazione di contaminazione $1 \mathrm{mg} / \mathrm{kg}$ ); infine, per quanto riguarda la concentrazione di Zn, nel poligono SP3 era di 346 mg/kg e nel poligono FS4 di $345 \mathrm{mg} / \mathrm{kg}$ (soglia di concentrazione di contaminazione $150 \mathrm{mg} / \mathrm{kg}$ ). Una volta definiti gli obiettivi della bonifica, sono state scelte due specie vegetali da utilizzare per il trattamento: "Heliantus annuиs" (girasole) e "Brassica juncea" (senape indiana). Prima di mettere in pratica il trattamento in situ, è stata valutata l'efficacia dello stesso mediante test di laboratorio e sistemi sperimentali in serra, dai quali si è evidenziata la necessità di effettuare dei lavaggi del sito mediante agenti chelanti per migliorare il tasso di rimozione dei contaminanti. Sulla base dei risultati ottenuti, sono state avviate le attività di bonifica in situ. Dopo alcune operazioni preliminari volte a rendere l'area adatta alle pratiche di Fitorisanamento, sono state seminate le specie vegetali con una densità di circa 8-9 piante a $\mathrm{m}^{2}$. È stato, inoltre, adoperato un sistema di microirrigazione per la distribuzione degli agenti chelanti, EDTA e Tiosolfato d'Ammonio TO, per favorire la mobilizzazione dei metalli. Per piantare le specie vegetali sono stati eseguiti due cicli colturali: Heliantus annus è stata piantata a inizio marzo e rimossa a fine agosto, mentre Brassica juncea è stata piantata a inizio settembre e rimossa ad inizio aprile. Al termine di ciascun ciclo colturale sono state eseguite analisi delle concentrazioni di $\mathrm{Cd}, \mathrm{Hg}$ e Zn presenti all'interno del suolo e assorbite dalle piante. Entrambe le specie vegetali hanno mostrato un incremento dell' assorbimento nei campioni raccolti dopo l'aggiunta degli agenti chelanti, avvenuta nel periodo di massima crescita delle piante e prima 
della fioritura. In particolare, nella biomassa di H. Annus, sono state riscontrate concentrazioni di Cd pari a $1,17 \mathrm{mg} / \mathrm{kg}$ per il poligono SP3 e 1,07 per FS4, concentrazioni di Hg di 0,27 nell'area SP3 e di 0,26 in FS4, e concentrazioni di Zn di $132 \mathrm{mg} / \mathrm{kg}$ per SP3 e di $112 \mathrm{mg} / \mathrm{kg}$ per la zona FS4. Anche B. Juncea ha mostrato un significativo accumulo di Cd, $\mathrm{Hg}$ e $\mathrm{Zn}$ : il contenuto di $\mathrm{Cd}$ è risultato pari a $1,28 \mathrm{mg} / \mathrm{kg}$ in SP3 e di $1,26 \mathrm{mg} / \mathrm{kg}$ nell' area $\mathrm{FS} 4$, il contenuto di $\mathrm{Hg}$ nella zona SP3 pari a $0,33 \mathrm{mg} / \mathrm{kg}$ e nell' area FS4 pari a $0,34 \mathrm{mg} / \mathrm{kg}$, e il contenuto di Zn pari a $144 \mathrm{mg} / \mathrm{kg}$ in SP3 e $140 \mathrm{mg} / \mathrm{kg}$ nel poligono FS4. I risultati delle analisi svolte, invece, all'interno del suolo trattato dopo il primo ciclo di coltura con H. Annus e il secondo con B. Juncea sono riportate in Fig. 2.

\section{Conclusioni}

Lo studio ha dimostrato che l'efficacia della Fitoestrazione di metalli pesanti (Cd, $\mathrm{Hg}, \mathrm{Zn}$ ) nelle colture testate aumenta se accoppiata a lavaggi del suolo mediante agenti chelanti e sostanze chimiche che influenzano la biodisponibilità dei contaminanti, aumentando la mobilità e l'assorbimento degli stessi. Entrambe le specie vegetali sono risultate eccellenti iperaccumulatrici di metalli pesanti, infatti sono state raggiunte percentuali di rimozione fino al $75 \%$ e in ogni caso, che hanno permesso di raggiungere l'obiettivo della bonifica, in quanto le concentrazioni di contaminanti sono risultate inferiori ai valori di concentrazione soglia di contaminazione Csc. Visto il breve periodo e i costi moderati, confrontabili con quelle di abituali pratiche agronomiche, con i quali tali risultati sono stati ottenuti, il fitorisanamento è certamente una tecnologia su cui si dovrà investire nel prossimo futuro. 


\section{References}

1.Glick, B. R. Using soil bacteria to facilitate phytoremediation. Biotechnology Advances 28 , 367-374 (2010).

2.Memon, A. R. \& Schröder, P. Implications of metal accumulation mechanisms to phytoremediation. Environmental Science and Pollution Research 16, 162-175 (2008).

3.L.Wang, B.Ji, Y.Hu, R.Liu \& W.Sun. A review on in situ phytoremediation of mine tailings. Chemosphere 184, 594-600 (2017).

4.D.K.Patra, C.Pradhan \& H.K.Patra. Toxic metal decontamination by phytoremediation approach: Concept challenges, opportunities and future perspectives. Environmental Technology \& Innovation 18, 100672 (2020).

5.V.Shah \& A.Daverey. Phytoremediation: A multidisciplinary approach to clean up heavy metal contaminated soil. Environmental Technology \& Innovation 18, 100774 (2020).

6.A.Mahar et al.. Challenges and opportunities in the phytoremediation of heavy metals contaminated soils: A review. Ecotoxicology and Environmental Safety 126, 111-121 (2016).

7.A.Ullah et al. Diazotrophs-assisted phytoremediation of heavy metals: a novel approach. Environmental Science and Pollution Research 22, 2505-2514 (2014).

8.Dary, M., Chamber-Pérez, M. A., Palomares, A. J. \& Pajuelo, E. "In situ" phytostabilisation of heavy metal polluted soils using Lupinus luteus inoculated with metal resistant plant-growth promoting rhizobacteria. Journal of Hazardous Materials 177, 323-330 (2010).

9.Guarino, C. \& Sciarrillo, R. The effectiveness and efficiency of phytoremediation of a multicontaminated industrial site: Porto Marghera (Venice Lagoon Italy). Chemosphere 183, 371-379 (2017). 


\section{Figure Captions}

Figure 1. Le piante come tecnica di bonifica

Figure 2. Concentrazioni di $\mathrm{Cd}, \mathrm{Hg}, \mathrm{Zn}$ nel terreno dopo i cicli di coltura 


\section{Figures}

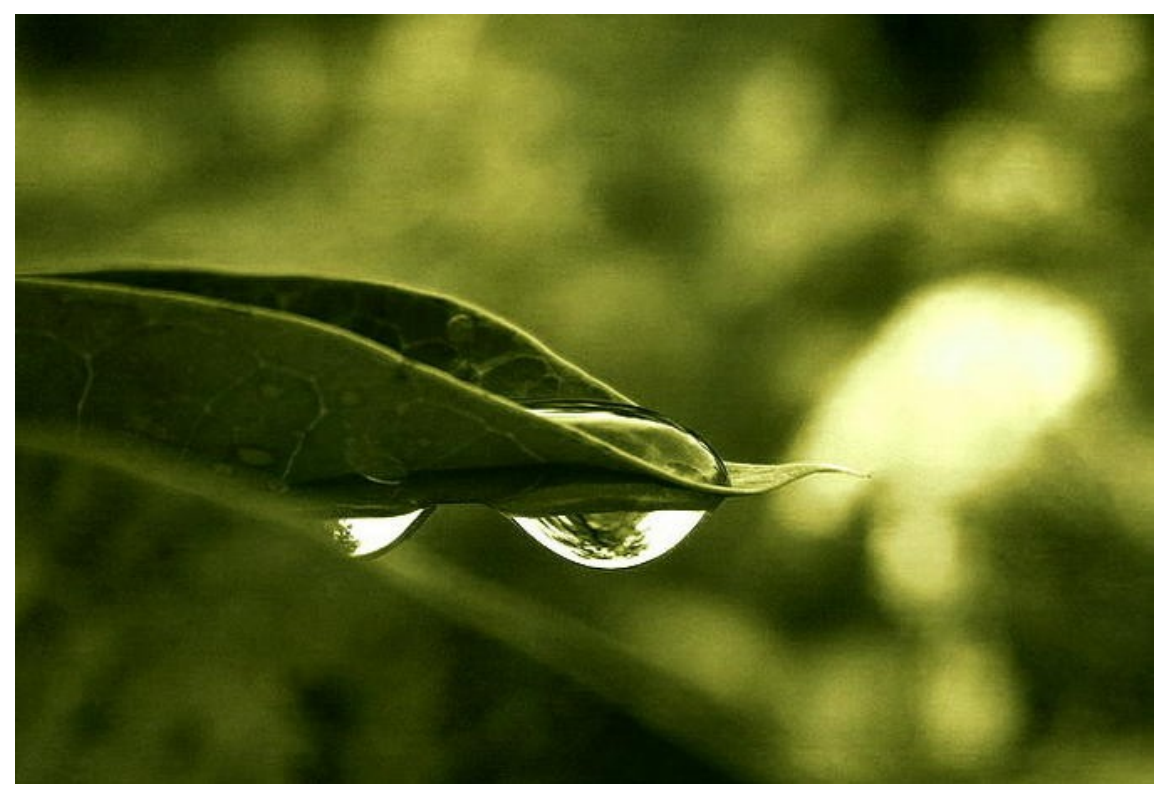

Figure 1: Le piante come tecnica di bonifica

\begin{tabular}{|l|l|l|l|l|}
\hline H. Annus & & & & \\
\hline Campione & UM & CD & Hg & Zn \\
\hline SP3 & $\mathrm{mg} / \mathrm{kg}$ & $2,23 \pm 0,04$ & $1,23 \pm 0,04$ & $214 \pm 12$ \\
\hline FS4 & $\mathrm{mg} / \mathrm{kg}$ & $2,17 \pm 0,04$ & $1,20 \pm 0,05$ & $234 \pm 13$ \\
\hline & & & & \\
\hline B. Juncea & & & & \\
\hline Campione & UM & CD & Hg & Zn \\
\hline SP3 & $\mathrm{mg} / \mathrm{kg}$ & $0,95 \pm 0,04$ & $0,86 \pm 0,04$ & $71,5 \pm 12$ \\
\hline FS4 & $\mathrm{mg} / \mathrm{kg}$ & $0,91 \pm 0,04$ & $0,85 \pm 0,05$ & $94 \pm 12$ \\
\hline
\end{tabular}

Figure 2: Concentrazioni di $\mathrm{Cd}, \mathrm{Hg}, \mathrm{Zn}$ nel terreno dopo i cicli di coltura 